\title{
Aspectos da história natural de Astyanax scabripinnis Jenyns (Teleostei, Characidae) em um riacho de floresta com araucária no sul do Brasil
}

\author{
Vinícius Abilhoa \\ Grupo de Pesquisas em Ictiofauna, Museu de História Natural Capão da Imbuia, Prefeitura de Curitiba. \\ Rua Professor Benedito Conceição 407, 82810-080 Curitiba, Paraná. E-mail: vabilhoa@uol.com.br
}

\begin{abstract}
Natural history aspects of Astyanax scabripinnis Jenyns (Teleostei, Characidae) in an Araucaria Forest stream in southern Brazil. In this paper the population structure, feeding habits and reproduction of Astyanax scabripinnis Jenyns, 1842 were described, based on the analysis of fish collected monthly from October 1998 to September 1999 in a forest stream of the upper Iguaçu River basin. Length frequency distributions registered were different between males $(5.4-6.7 \mathrm{~cm})$ and females $(6.1-8.1 \mathrm{~cm})$. Significant differences of condition factor values between sexes were found, meaning different growth rates. The reproductive period occurred from October to March, as described for other characids of the same area. Terrestrial insects fragments, superior vegetables residue and aquatic insects larvae were the main food items consumed, indicating a pronounced dependence of the feeding on the allochthonous items imported from the riparian vegetation. Possible implications of physical, chemical and hydrologic seasonal variations on A. scabripinnis natural history were discussed. KEY WORDS. Biological parameters; feeding; population structure.
\end{abstract}

RESUMO. Neste trabalho é descrita a estrutura populacional, os hábitos alimentares e a reprodução de Astyanax scabripinnis Jenyns, 1842, baseado em coletas realizadas mensalmente entre outubro de 1998 e setembro de 1999, em um riacho das cabeceiras do rio Iguaçu. A distribuição de freqüiência por classe de comprimento revelou valores diferentes entre machos $(5,4-6,7 \mathrm{~cm})$ e fêmeas $(6,1-8,1 \mathrm{~cm})$. Foram encontradas diferenças significativas para os valores do fator de condição entre os sexos, o que pode implicar em diferentes taxas de crescimento. $O$ período reprodutivo ocorreu entre outubro e março, como já descrito para outros caracídeos da mesma região. Fragmentos de insetos terrestres, restos de vegetais superiores e larvas de insetos aquáticos foram os principais itens consumidos, indicando uma pronunciada dependência da alimentação com os itens alóctones importados da vegetação ripária. Possíveis implicações das variações sazonais dos parâmetros físicos, químicos e hidrológicos sobre a história natural de $A$. scabripinnis são discutidas.

PALAVRAS-CHAVE. Alimentação; estrutura populacional; parâmetros biológicos.

Estudos realizados em pequenos cursos de água têm demonstrado que as comunidades destes ecossistemas aquáticos são submetidas constantemente a mudanças nas condições hidrodinâmicas (MATTHEws 1998), principalmente em função da pluviosidade e de fatores abióticos associados (Peres Neto et al. 1995, Bizerril 1997). Para os peixes, estas mudanças estacionais decorrentes da expansão e contração do ambiente aquático durante as variações climáticas podem influenciar a alimentação, reprodução e tamanho das populações (Welcomme 1969, Matthews 1982, Meffe \& Sheldon 1988, Angermeier 1989, CASATTI 2005), fato que lhes confere complexidade no estudo e conseqüente conservação.

Na bacia do rio Iguaçu, os cursos de água relativamente pequenos são ambientes favoráveis à propagação de inúmeras espécies de peixes, sendo a família Characidae uma das mais diversificadas. Dentro deste grupo destacam-se os lambaris do gênero Astyanax, o qual compreende pelo menos dez espécies na bacia hidrográfica, muitas das quais ainda não descritas (Severi \& Cordeiro 1995, Garavello et al. 1997, Ingenito et al. 2004).

Nos últimos anos, as diagnoses das espécies de Astyanax Baird \& Girard, 1854 que ocorrem em pequenos riachos de diferentes bacias hidrográficas brasileiras têm-se defrontado com uma combinação de caracteres, às quais o nome Astyanax scabripinnis Jenyns, 1842 tem sido atribuído (Melo 2001, Shibatta et al. 2002, Castro et al. 2003, Bennemann et al. 2005, Oyakawa et al. 2006). Esta denominação segue o sentido amplo de $A$. scabripinnis de EIgENMANN (1921, 1927), sendo os seguintes caracteres diagnósticos considerados: altura do corpo entre 2,6 e 3,6 vezes no comprimento padrão, 25 ou menos raios na nadadeira anal, cabeça robusta e corpo também mais robusto na altura das nadadeiras peitorais. 
Entretanto, o nome Astyanax scabripinnis refere-se provavelmente a um complexo de espécies (Moreira-Filho \& Bertollo 1991), o qual necessita de uma ampla revisão e de uma melhor definição taxonômica e biológica (BERTACo \& Malabarba 2001, Bertaco \& Lucena 2006), sendo que a população aqui estudada pode representar uma nova espécie relacionada a este complexo.

Estudos realizados com A. scabripinnis indicam a preferência da espécie por rios de menor porte (Castro \& CasatTi 1997, Lemes \& Garutti 2002, Casatti 2002, Shibatta et al. 2002, Veregue \& ORSi 2003, BENNEMANN et al. 2005), onde os indivíduos consomem uma grande quantidade de recursos alóctones (SніватTA et al. 2002, Bennemann et al. 2005). Este fato parece estar diretamente relacionado com a capacidade de muitas espécies do gênero Astyanax em colonizar diferentes habitats (Esteves \& GALETTI 1995, HaHn et al. 1998, Vilella et al. 2002, OrSi et al. 2004).

O presente estudo teve como objetivo a obtenção de informações a respeito da estrutura populacional, alimentação e reprodução de uma população de Astyanax scabripinnis em um riacho localizado nas cabeceiras do rio Iguaçu, que atualmente corresponde a área de influência direta do Reservatório do Iraí. Estas informações sobre a história natural do grupo devem contribuir para a elaboração de estratégias de conservação deste novo ecossistema, visando a conservação da espécie.

\section{MATERIAL E MÉTODOS}

\section{Área de estudo}

A área de estudo está situada na Região Metropolitana de Curitiba, com seus limites entre as coordenadas $25^{\circ} 21^{\prime} 40^{\prime \prime}$ e $25^{\circ} 26^{\prime} 50^{\prime \prime}$ de latitude sul e $49^{\circ} 08^{\prime} 30^{\prime \prime}$ e $49^{\circ} 01^{\prime} 05^{\prime \prime}$ de longitude oeste. Corresponde atualmente à área de influência direta do Reservatório do Iraí, que teve sua construção finalizada no final de 1999 e primeiro extravasamento em janeiro de 2001 (BolLmann et al. 2005).

Com um relevo suavemente ondulado, a região apresentava uma área de drenagem com pequenos rios, como o Curralinho, Timbú, Cerrado e Canguiri, os quais formam o rio Iraí, que faz parte da rede hidrográfica que dá origem a bacia hidrográfica do rio Iguaçu. $\mathrm{Na}$ área de estudo, o rio Iraí apresentava pouca profundidade, com correnteza moderada e largura de até três metros. Seu substrato era formado principalmente por areia e resíduos vegetais, com as encostas compostas de argila e areia.

Antes da formação do reservatório, a floresta de galeria que acompanhava o rio Iraí sofria inundações freqüentes (Floresta Ombrófila Mista Aluvial), que proporcionavam deposição aluvial nestes períodos, denotando o caráter edáfico desta formação de Floresta com Araucária. A várzea que ladeava o rio apresentava-se coberta por espécies herbáceas e arbustivas.

Muito embora o comportamento das espécies aquáticas frente aos fatores físicos e químicos deste ambiente ainda não seja conhecido, é provável que, como já descrito para áreas naturalmente alagáveis, a flutuação nos fatores ambientais duran- te as inundações da floresta de galeria exerça grande influência sobre a disponibilidade de alimento e abrigo para a fauna aquática (Esteves 1996, Harper et al. 1997).

\section{Procedimentos amostrais}

Os exemplares de Astyanax foram obtidos através de amostragens mensais padronizadas ( 24 horas) que foram realizadas entre outubro de 1998 e setembro de 1999 no rio Iraí. Para as coletas foram utilizadas redes de espera, com malhas simples de tamanhos 1,5 e $2,5 \mathrm{~cm}$ entre nós consecutivos e tarrafas, com malhas simples de $1,5 \mathrm{~cm}$. Para coleta de exemplares pequenos foram utilizadas peneiras e puçás, de forma sistematizada, com duração de 30 minutos em cada coleta mensal. Os espécimes amostrados foram colocados em sacos plásticos etiquetados contendo solução de formol $4 \%$.

Em laboratório os exemplares foram identificados, contados e mensurados para a obtenção do comprimento total e padrão, pesados (com auxílio de balança de precisão de centésimo de grama) e dissecados para a remoção do trato digestório e determinação do sexo e estádio de maturação gonadal segundo escala proposta por VAzzoler (1996). A determinação do estádio de maturação foi baseada no tamanho dos exemplares e em características como coloração e volume das gônadas, grau de turgidez, transparência, irrigação sanguínea e presença, no caso dos ovários, de ovócitos visíveis a olho nu. Alguns exemplares foram tombados na Coleção de Peixes do Museu de História Natural Capão da Imbuia (MHNCI 10374).

Durante cada amostragem mensal, dados físicos e químicos de temperatura do ambiente, temperatura da água, vazão média $\left(\mathrm{m}^{3} / \mathrm{s}\right)$, potencial hidrogeniônico $(\mathrm{pH})$ e oxigênio dissolvido (OD) foram obtidos da Rede de Monitoramento da SUDERHSA/PR para o trecho estudado. Dados de precipitação utilizados para a caracterização histórica dos padrões de chuvas na área foram fornecidos pelo SIMEPAR, abrangendo o período de fevereiro de 1970 até dezembro de 1999.

\section{Análise dos dados}

Para a análise dos dados de estrutura populacional em tamanho, a distribuição de freqüência das classes de comprimento total para ambos os sexos foi obtida pela regra de Sturge (Vieira 1980).

A relação entre o peso e o comprimento total foi obtida para machos e fêmeas das espécies analisadas, sendo expressa pela equação: Peso = K x Comprimento total ${ }^{\mathrm{b}}$. Os parâmetros $\mathrm{K}$ e $\mathrm{b}$ foram obtidos ajustando-se o logaritmo das variáveis dependente (pesos) e independente (comprimentos totais) pelo método dos mínimos quadrados (Santos 1978, VAzzoler 1981). O valor b é a constante de crescimento relativo, e tende a assumir valores próximos a 3, quando o crescimento é isométrico (Weatherley \& Gill 1987).

$\mathrm{O}$ valor de $\mathrm{K}$ é denominado de fator de condição, e pode ser utilizado para a avaliação de respostas fisiológicas entre indivíduos de distintos tamanhos e variados estágios de desenvolvimento, sendo que diferenças encontradas são atribuídas às con- 
dições gerais do organismo (CHAPMAn et al. 1977, WiLnNer et al. 1979, VAzZoler 1981). Os valores do fator de condição foram calculados para cada indivíduo amostrado através da equação: $\mathrm{K}$ $=$ Peso/Comprimento total ${ }^{\mathrm{b}}$ (SANTos 1978, VAzzoler 1981), sendo que as diferenças entre os valores médios para cada parâmetro considerado foram avaliadas pelo teste de Kruskall-Wallis (VIEIRA 2003), tendo em vista que os valores não apresentaram distribuição normal segundo o teste de Shapiro-Wilk.

A proporção sexual foi determinada através das distribuições mensais das freqüências de machos e fêmeas ao longo do período de estudo. Utilizou-se o teste de "qui-quadrado" $\left(\chi^{2} ; \alpha=0,05\right)$ para a verificação de eventuais diferenças significativas nestas proporções (Zar 1996). A distribuição de freqüência dos estádios de maturação gonadal foi utilizada para determinar o período reprodutivo (VAzzoler 1981).

$\mathrm{O}$ trato digestório de 15 exemplares em cada coleta foi removido e fixado em solução de formol a $4 \%$, sendo posteriormente conservado em solução de álcool a 70\%. Os conteúdos estomacais foram analisados sob microscópio estereoscópico, sendo que a identificação dos itens foi realizada com auxílio de bibliografia especializada (Pérez 1988, Borror et al. 1989) e através da consulta a especialistas (Laboratório de Ecologia Bêntica - MHNCI).

Para a análise da importância dos itens alimentares identificados foi utilizado o método de freqüência de ocorrência, que avalia percentual de estômagos em que determinado item alimentar ocorre e o método de pontos, onde a contribuição de cada item é determinada pela proporção de quadrículas ocupadas pelo item em uma superfície plana quadriculada em relação ao número total de quadrículas ocupadas pelo conteúdo (Hynes 1950, Hyslop 1980). Para análise da importância efetiva de cada item na alimentação da espécie foi utilizado o Índice Alimentar (IAi) proposto por KAWAKAMI \& VAZZOLER (1980).

O padrão de similaridade entre as dietas foi obtido através de uma análise de agrupamento da matriz do IAi dos itens alimentares em cada estação do ano, utilizando-se o coeficiente de distância euclidiana e o critério de agrupamento pelo método de Ward. Esse coeficiente representa a menor distância geométrica entre os grupos analisados em um espaço multidimensional, e o método de variâncias mínimas (Ward) utiliza a análise de variância na avaliação das distâncias entre os grupos, tentando sempre minimizar a soma dos quadrados. As análises foram realizadas através do programa MVSP 3.1 (Kovach Comp. Services 1999).

A tabulação dos dados, testes estatísticos e a confecção dos gráficos foi realizada com auxílio de software Statistica 5.1 (StatSoft Inc. 1997) e MS Excel 9.0 (Microsoft Corporation 1999).

\section{RESULTADOS}

\section{Condições abióticas}

Os padrões de precipitação na área, estabelecidos com base nos dados pluviométricos históricos (29 anos) revela um valor médio de $1.415 \mathrm{~mm}$, com mínimas de $277 \mathrm{~mm}$ e a máxi- ma média de $3.214 \mathrm{~mm}$. As chuvas concentram-se nos meses de dezembro e janeiro, com valores médios de 157 e 189 mm, respectivamente. Os meses de menor precipitação média foram abril, com $79 \mathrm{~mm}$ e agosto, com $72 \mathrm{~mm}$ (Fig. 1).

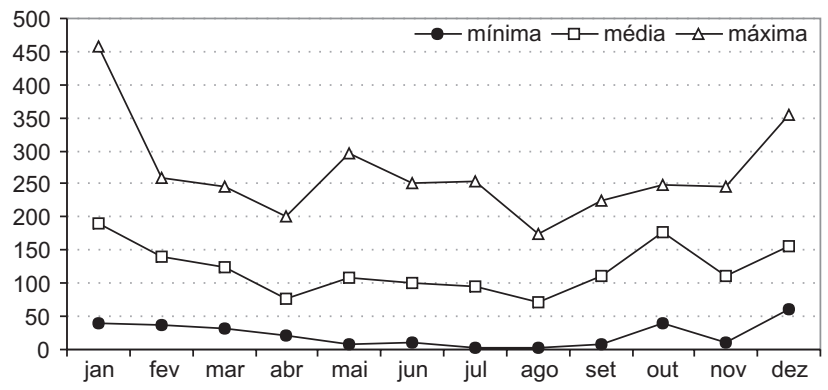

Figura 1. Distribuição mensal das precipitações mínima, média e máxima na região do alto rio Iguaçu, considerando-se as médias mensais entre 1970 a 1999. Dados do Simepar.

A temperatura e as concentrações de oxigênio dissolvido (OD) variaram ao longo do período amostral, sendo os maiores valores de temperatura observados no verão, período de maior precipitação. Para o OD, os maiores valores foram registrados entre o outono e o inverno. Os valores de $\mathrm{pH}$ apresentaram pequenas variações entre as quatro estações do ano, apresentando-se próximos à neutralidade, com oscilações entre 6,2 e 7,5 (Tab. I).

A série de descargas médias mensais obtida para o local de estudo, mostrada na tabela I, apresenta uma vazão média de

Tabela I. Variações das condições abióticas na área de estudo, caracterizadas por um único registro mensal dos dados físicos e químicos de temperatura do ambiente, temperatura da água, vazão média $\left(\mathrm{m}^{3} / \mathrm{s}\right)$, potencial hidrogeniônico $(\mathrm{pH})$ e oxigênio dissolvido (OD), rio Iraí, Paraná, outubro de 1998 a setembro de 1999.

\begin{tabular}{lccccc}
\hline \multicolumn{1}{c}{ Mês } & $\begin{array}{c}\text { Temperatura do Temperatura } \\
\text { ambiente }\left({ }^{\circ} \mathrm{C}\right)\end{array}$ & $\begin{array}{c}\text { Vazão } \\
\text { da água }\left({ }^{\circ} \mathrm{C}\right)\end{array}$ & $\begin{array}{c}\mathrm{pH} / \mathrm{s}) \\
\mathrm{pH}\end{array}$ & $\begin{array}{c}\mathrm{OD} \\
(\mathrm{mg} / \mathrm{l})\end{array}$ \\
\hline Outubro & 18,5 & 16,0 & 2,23 & 6,2 & 6,9 \\
Novembro & 19,0 & 17,0 & 2,06 & 7,0 & 5,5 \\
Dezembro & 20,0 & 17,5 & 2,18 & 7,2 & 6,7 \\
Janeiro & 23,5 & 19,0 & 2,74 & 7,3 & 6,8 \\
Fevereiro & 26,0 & 21,5 & 2,97 & 6,9 & 6,6 \\
Março & 21,5 & 17,0 & 2,42 & 7,5 & 7,2 \\
Abril & 21,0 & 19,0 & 1,85 & 6,6 & 6,2 \\
Maio & 17,0 & 17,0 & 1,87 & 6,8 & 8,3 \\
Junho & 14,5 & 10,5 & 1,85 & 7,2 & 9,2 \\
Julho & 16,0 & 13,0 & 1,88 & 7,2 & 7,9 \\
Agosto & 19,0 & 16,0 & 1,60 & 6,5 & 7,9 \\
Setembro & 21,5 & 18,5 & 1,96 & 7,0 & 8,1 \\
\hline
\end{tabular}

Fonte: Rede de Monitoramento da SUDERHSA, Governo do Paraná. 
longo período de $2,14 \mathrm{~m}^{3} / \mathrm{s}$, com valores maiores para o verão, período de maior precipitação. A vazão máxima média mensal observada no período de estudo ocorreu em fevereiro, com um valor de $2,97 \mathrm{~m}^{3} / \mathrm{s}$, enquanto que a vazão média mensal mínima ocorreu em agosto, com um valor de 1,60 m³ $/ \mathrm{s}$.

\section{Estrutura das populações}

Foram consideradas sete classes de comprimento total para os 217 peixes capturados, 140 fêmeas e 77 machos, e a amplitude de tamanho variou da classe 4,7-5,3 cm à classe 8,9$9,5 \mathrm{~cm}$. Indivíduos menores que $4,7 \mathrm{~cm}$ não foram coligidos. A distribuição de freqüência por classe de comprimento total revelou que os valores modais foram diferentes entre os sexos, sendo os machos mais abundantes nas classes de comprimento de 5,4-6,7 cm e fêmeas entre 6,1-8,1 cm (Fig. 2).

A distribuição de comprimento total por classes revelou também que as capturas de machos foram mais freqüentes na classe de 6,1-6,7 cm e fêmeas na classe 6,8-7,4 cm (Fig. 2).

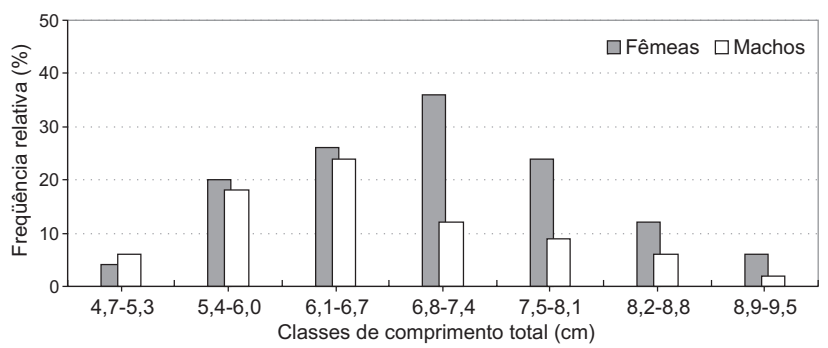

Figura 2. Distribuição de freqüência por classe de comprimento de machos e fêmeas de Astyanax scabripinnis capturados na área estudada no rio Iraí, Paraná, entre os anos de 1998 e 1999.

A relação peso e comprimento para machos e fêmeas de Astyanax estão representados na figura 3, assim como suas expressões matemáticas. Verifica-se que o valor do parâmetro $b$ foi de 3,1 para machos e 2,9 para fêmeas.

Como os valores do fator de condição não apresentaram distribuição normal para os exemplares machos (Shapiro-Wilk $\mathrm{W}$ teste $=0,956 ; \mathrm{p}<0,05$ ) e fêmeas de Astyanax (Shapiro-Wilk $\mathrm{W}$ teste $=0,977 ; \mathrm{p}<0,05)$, as médias foram avaliadas através do teste não-paramétrico de Kruskall-Wallis, sendo que os limites de confiança foram comparados para a identificação de diferenças significativas.

Foram encontradas diferenças significativas para os valores do fator de condição entre machos e fêmeas $(H=139,52$; $\mathrm{p}<0,05)$, sendo que as fêmeas apresentaram médias maiores. Com relação às variações sazonais desse parâmetro, machos $(\mathrm{H}=22,45 ; \mathrm{p}<0,05)$ e fêmeas $(\mathrm{H}=11,01 ; \mathrm{p}<0,05)$ apresentaram diferenças significativas entre as estações do ano, com valores médios menores no inverno e maiores no período primavera/verão.

A proporção sexual durante o período de estudo está representada na figura 4. Para Astyanax observou-se um predo-
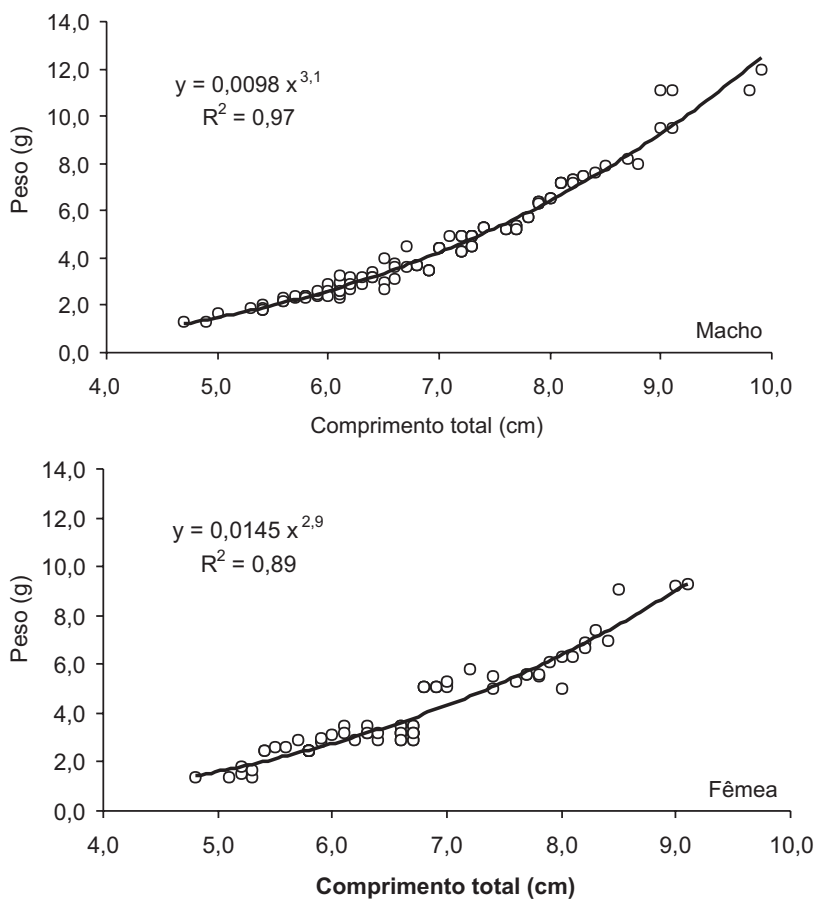

Figura 3. Diagrama de dispersão, linha de tendência, coeficiente de correlação de Pearson e equação de relacionamento entre as variáveis peso/comprimento total (CT) para exemplares de Astyanax scabripinnis capturados mensalmente na área estudada no rio Iraí, Paraná, entre os anos de 1998 e 1999.

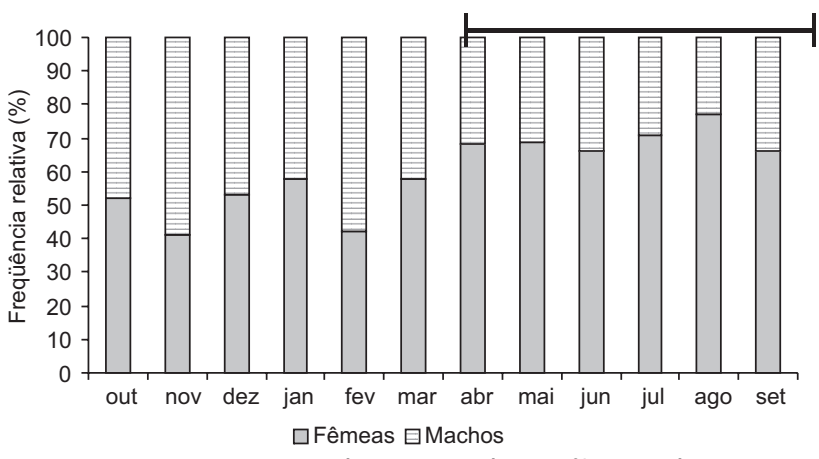

Figura 4. Proporção mensal entre machos e fêmeas de Astyanax scabripinnis capturados na área estudada no rio Iraí, Paraná, nos anos de 1998 e 1999. A barra horizontal indica diferenças significativas.

mínio significativo de fêmeas durante o outono e o inverno, nos meses de abril $\left(\chi^{2}=13,2 ; \mathrm{p}<0,05\right)$, maio $\left(\chi^{2}=12,5 ; \mathrm{p}<\right.$ $0,05)$, junho $\left(\chi^{2}=10,9 ; \mathrm{p}<0,05\right)$, julho $\left(\chi^{2}=12,4 ; \mathrm{p}<0,05\right)$, agosto $\left(\chi^{2}=21,5 ; \mathrm{p}<0,05\right)$ e setembro $\left(\chi^{2}=16,3 ; \mathrm{p}<0,05\right)$ de 1999. Entre outubro de 1998 e março de 1999 não foram observadas diferenças nas freqüências de machos e fêmeas. 
A distribuição de freqüência mensal dos estádios de desenvolvimento gonadal está representada na figura 5. Gônadas maduras e indivíduos imaturos ocorreram em todo período amostral, com maiores freqüências entre outubro de 1998 e abril de 1999 (primavera-outono).

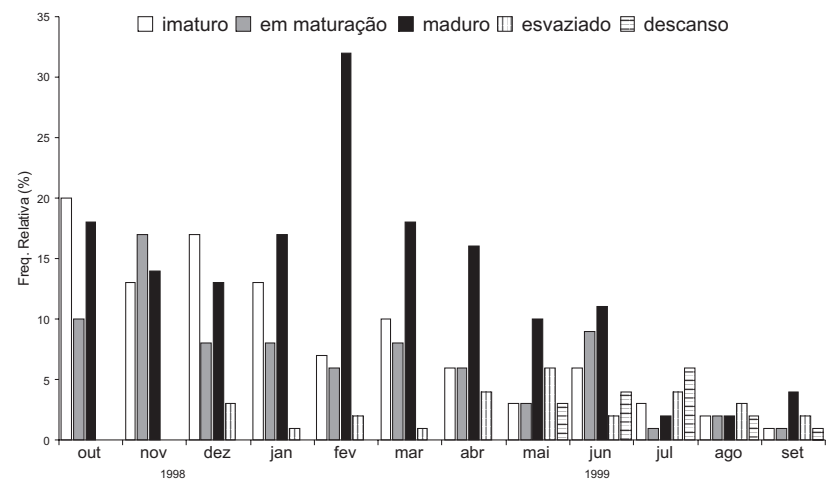

Figura 5. Freqüência relativa mensal dos estádios de maturação de Astyanax scabripinnis capturados na área estudada no rio Iraí, Paraná, nos anos de 1998 e 1999.

Um total de 16 itens alimentares foi identificado nos estômagos de 120 exemplares, sendo que os itens mais importantes foram insetos e restos de vegetais superiores (Tab. II).

Durante a primavera e o verão foram observadas as maiores diversidades de itens alimentares, com uma contribuição diferenciada de insetos alóctones e autóctones.

A análise de similaridade da dieta entre as estações do ano, permitiu estabelecer dois grupos distintos. O primeiro foi caracterizado pelo outono e verão, que compartilharam cinco itens alimentares (45,4\% do total entre essas estações), sendo as maiores proporções de fragmentos de insetos, restos vegetais e larvas de Odonata. O segundo agrupamento envolveu o inverno e a primavera, que compartilharam quatro itens $(36,3 \%$ do total entre essas estações), com contribuições relevantes de fragmentos de insetos, restos vegetais e larvas de Chironomidae (Fig. 6).

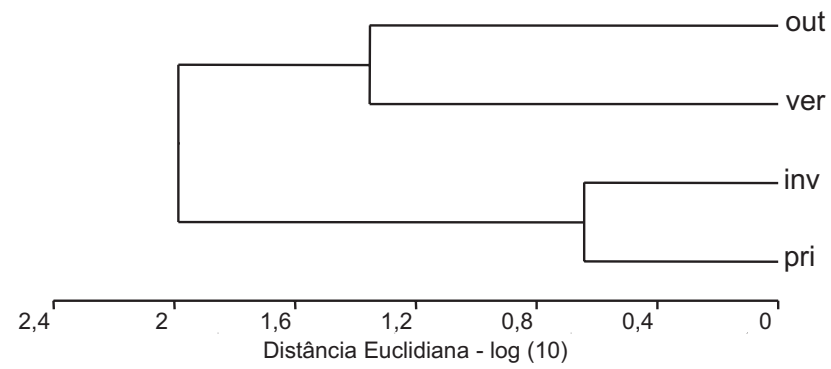

Figura 6. Dendrograma representando a similaridade (Distância Euclidiana) na dieta de Astyanax scabripinnis entre as estações do ano na área estudada no rio Iraí, Paraná, nos anos de 1998 e 1999.

\section{DISCUSSÃO}

Embora o termo "ictiofauna de riachos" não possa definir uma unidade natural (Buckup 1999), a predominância de alguns padrões, como o pequeno porte de seus componentes (CASTro 1999) e as adaptações às mudanças estacionais (LowEMcConnell 1975), permite a caracterização desse ecossistema particular, o qual apresenta uma série de características fisiográficas e hidrológicas que influenciam diretamente os ciclos de vida das espécies que aí vivem (Matrhews 1998).

$\mathrm{Na}$ área de estudo, as variáveis físico-químicas e hidrológicas apresentaram variações sazonais relevantes, com possíveis implicações sobre a composição da ictiofauna, como já constatado por diversos autores (UIEDA 1984, TAYLOR 1996, Takeuti et al. 1999, Lemes \& Garutti 2002). Nos meses de pluviometria mais intensa, quando os pulsos de vazão e os valores de temperatura são maiores, as mudanças podem ter atuação mais acentuada, dada a dimensão reduzida desses ambientes. Essa associação de fatores pode indicar que os riachos de cabeceira são ambientes instáveis, e que a ictiofauna apresenta diferentes adaptações como resultado dessas mudanças (MatThews 1998).

O lambari Astyanax scabripinnis apresentou variações nos parâmetros populacionais estudados, o que deve estar relacionado às alterações hidrológicas impostas pelo regime sazonal. As fêmeas foram registradas em número mais elevado, com exceção na classe de tamanho 4,7-5,3 cm, sendo que este fato já foi constatado por muitos autores (Gomes \& MonTeIro 1955, Gurgel 1995, Schifino et al. 1998, TAKeuti et al. 1999), inclusive para Astyanax (Nomura 1975), o que pode estar relacionado a diversos fatores, como taxas de crescimento diferenciado, maior mortalidade dos machos, maior longevidade das fêmeas ou até mesmo à suscetibilidade aos aparelhos de pesca utilizados.

Desconsiderando-se a seletividade do método de captura, a idéia de taxas de crescimento diferenciadas entre os sexos parece bastante plausível, tendo em vista as diferenças observadas na estrutura em tamanho e nos parâmetros da relação entre o peso e o comprimento total. Embora machos e fêmeas tenham apresentado crescimento praticamente isométrico, com os valores do coeficiente angular próximos a três, o fator de condição apresentou diferenças significativas entre os sexos e as estações do ano. De acordo com Le Cren (1951), essas diferenças podem ser interpretadas como medidas de várias características biológicas, podendo estar relacionadas ao peso das gônadas (período reprodutivo) (Benedito-Cecílio \& Agostinho 1997).

A proporção sexual somente não apresentou diferenças durante o período reprodutivo, o qual foi semelhante ao observado para outras espécies na bacia do Paraná (VAzzoler \& Menezes 1992, Vazzoler et al. 1997) e no reservatório de Segredo no médio rio Iguaçu (Suzuki \& Agostinho 1997). A reprodução parece ter ocorrido com maior freqüência entre outubro e março, período de maior pluviosidade, sendo que esta estratégia pode refletir adaptações dessa espécie às condições ambientais locais (BARBIERI \& BARBIERI 1988). Esta relação, entretanto, neces- 
Tabela II. Valores do índice de importância alimentar e da freqüência de ocorrência, IAi(FO), dos itens alimentares registrados no conteúdo estomacal de Astyanax scabripinnis capturados no rio Iraí, Paraná, entre outubro de 1998 e setembro de 1999. (AU) Itens autóctones, $(\mathrm{AL})$ itens alóctones, $(\mathrm{I})$ itens de procedência incerta.

\begin{tabular}{|c|c|c|c|c|c|}
\hline Itens alimentares & Itens & Primavera & Verão & Outono & Inverno \\
\hline Acarina & $\mathrm{I}$ & $0,03 \quad(2,4)$ & & & \\
\hline Araneae & $\mathrm{AL}$ & $0,13 \quad(2,4)$ & & & \\
\hline Aegla sp. (Crustacea, Decapoda) & $\mathrm{AU}$ & $0,18 \quad(3,1)$ & & & \\
\hline Copepoda & $\mathrm{AU}$ & $3,10 \quad(8,1)$ & $0,50 \quad(4,1)$ & & \\
\hline Larvas de Chironomidae (Insecta, Diptera) & $\mathrm{AU}$ & $15,3(42,0)$ & $0,90(22,5)$ & $3,70(13,1)$ & $1,20 \quad(9,4)$ \\
\hline Larvas de Simulidae (Insecta, Diptera) & $\mathrm{AU}$ & $0,30 \quad(2,4)$ & & & \\
\hline Diptera (Insecta) & $\mathrm{AL}$ & & & $4,40(18,1)$ & $0,80 \quad(1,9)$ \\
\hline Larvas de Odonata (Insecta) & $\mathrm{AU}$ & & $12,1(23,2)$ & $12,2(43,1)$ & \\
\hline Larvas de Trichoptera (Insecta) & $\mathrm{AU}$ & & $15,5(18,5)$ & & \\
\hline Larva de Lepidoptera (Insecta) & $\mathrm{AL}$ & & $0,01 \quad(2,7)$ & $3,90 \quad(9,1)$ & \\
\hline Elmidae (Insecta, Coleoptera) & $\mathrm{AL}$ & $0,56(12,3)$ & & & \\
\hline Cerambicidae (Insecta, Coleoptera) & $\mathrm{AL}$ & & $0,50 \quad(2,7)$ & & \\
\hline Formicidae (Insecta, Hymenoptera) & $\mathrm{AL}$ & $0,50 \quad(2,4)$ & $0,09 \quad(2,7)$ & & $0,19 \quad(2,3)$ \\
\hline Vellidae (Insecta, Heteroptera) & AL & & $0,01 \quad(2,7)$ & & \\
\hline Fragmentos de insetos terrestres & $\mathrm{AL}$ & $70,8(89,1)$ & $58,6(65,3)$ & $62,7(75,8)$ & $85,5(73,5)$ \\
\hline Restos de vegetais superiores & $\mathrm{AL}$ & $9,10(29,1)$ & $11,8(19,3)$ & $13,1(29,3)$ & $12,3(29,4)$ \\
\hline
\end{tabular}

sita ser melhor investigada, visto que a pluviosidade pode apresentar interações com outras variáveis ambientais.

Com relação à alimentação, estudos sobre a dieta de peixes tropicais de água doce mostram que, de maneira geral, os peixes apresentam uma considerável plasticidade trófica (LowEMcConnell 1999), sendo quase sempre euritróficos (AвelHa et al. 2001), ou seja, mudam sua dieta de acordo com variações espaciais e temporais na disponibilidade dos itens alimentares. Como os riachos possuem uma produtividade primária relativamente baixa e os processos são predominantemente heterotróficos e de carreamento, a ictiofauna destes ambientes depende da importação da matéria alóctone para a sua subsistência (CASTro 1999). Assim, nesses sistemas, as matas ciliares exercem grande importância no que se refere à fonte de alimentos, composição de hábitats e refúgios, que são fatores importantes para manutenção da diversidade da fauna aquática (Crowder \& Cooper 1982, Gilinsky 1984, Gotceitas \& Colgan 1989, Vono \& Barbosa 2001).

Além de maior diversidade, a vegetação ripária permite a existência na comunidade de peixes de elementos com maior grau de especialização (Rozas \& Odum 1988, Greenberg 1991, Johnson \& Jennings 1998, Lowe-Mcconnell 1999, Wootton 1999), pois possibilita o uso de frutos, folhas e flores como alimento de várias espécies (LowE-McConNell 1999), assim como artrópodes terrestres que caem da vegetação ciliar e larvas aquáticas de insetos que se alimentam, direta ou indiretamente, deste material orgânico alóctone importado da vegetação (CASTRO 1999).
Essa flexibilidade alimentar dos peixes de riachos aumenta as chances de sobrevivência das espécies, principalmente em locais com eventual escassez de recursos alimentares (BARRELLA et al. 2001), sendo que esta considerável plasticidade na dieta pode ser atribuída às alterações no ambiente (LowE-McCONNELL 1999, АвеLHA et al. 2001).

Estas afirmações são concordantes com os resultados obtidos no presente estudo, sendo que o hábito alimentar registrado se assemelha aos observados para outros representantes do gênero nesse mesmo tipo de ecossistema (UiEDA 1984, Costa 1987, Sabino \& Castro 1990, Sabino \& Zuanon 1998, CasatTi \& Castro 1998, Bennemann et al. 2005).

Com base nos resultados foi possível observar que os fragmentos de insetos (terrestres - alóctones) e os restos de vegetais superiores seguidos de larvas de insetos aquáticos compõem a base da dieta da espécie, independentemente da estação do ano considerada. Este hábito onívoro, com tendência à insetivoria, esteve provavelmente relacionado à captura de muitos exemplares em remansos cobertos por densa vegetação pendente sobre o riacho, a qual forneceu recursos alóctones para a ictiofauna estudada.

A importância deste recurso para as comunidades que compõe os ambientes de riachos localizados em áreas com mata ciliar preservada tem sido constantemente registrada (CASATTI 2002, Mazzoni \& Rezende 2003, Rezende \& Mazzoni 2006). No caso do lambari A. scabripinnis, estudos realizados indicam que a espécie apresenta hábito alimentar insetívoro, com predomí- 
nio de insetos terrestres - alóctones na dieta (Sніватта et al. 2002, Bennemann et al. 2005), resultados estes também constatados no riacho aqui estudado.

As pequenas divergências nas contribuições percentuais dos itens, apesar da grande similaridade sazonal da dieta, e a maior variedade de presas em alguma estação do ano podem ser atribuídas a diferenças na disponibilidade de alimento, visto que o regime estacional moderado da região permite a divisão em um período chuvoso, com temperaturas mais altas, e outro parcialmente seco, com temperaturas médias mais baixas.

Os resultados deste estudo apontam para a importância da manutenção dos riachos de cabeceiras na bacia do alto rio Iguaçu, visto que os fenômenos que ocorrem neste tipo de ecossistema aquático determinam alterações no ciclo biológico de diversas espécies que utilizam esses ambientes tão singulares, como o caso de Astyanax scabripinnis.

\section{REFERÊNCIAS BIBLIOGRÁFICAS}

Abelha, M.C.F.; A.A. Agostinho \& E. Goulart. 2001. Plasticidade trófica em peixes de água doce. Acta Scientiarum 23: 425434.

Angermeier, P.L. 1989. Species-area relationships for stream fishes. Ecology 70 (5): 1450-1462.

BARbieri, G. \& M.C. BARbieri. 1988. Curva de maturação, tamanho de primeira maturação gonadal e fecundidade de Astyanax bimaculatus e Astyanax fasciatus da represa do Lobo, Estado de São Paulo (Osteichthyes, Characidae). Ceres 35 (197): 64-77.

Barrella, W.; M. Petrere; W.S. Smith \& L.F.A. Montag. 2001. As relações entre as matas ciliares, os rios e os peixes, p.187207. In: R.R. Rodrigues \& H.F. LeITão (Eds). Matas ciliares: conservação e recuperação. São Paulo, Edusp, 320p.

Benedito-Cecílio, E. \& A.A. Agostinho. 1997. Estrutura das populações de peixes do reservatório de Segredo, p. 113-139. In: A.A. Agostinho \& L.C. Gomes (Eds). Reservatório de Segredo: bases ecológicas para o manejo. Maringá, EDUEM, 387p.

Bennemann, S.T.; A.M. Gealh; M.L. Orsi \& L.M. de Souza. 2005. Ocorrência e ecologia trófica de quatro espécies de Astyanax (Characidae) em dieferentes rios da bacia do rio Tibagi, Paraná, Brasil. Iheringia, Série Zoologia, 95 (3): 247-254.

Bertaco, V.A. \& C.A.S. Lucena. 2006. Two new species of Astyanax (Ostariophysi: Characiformes: Characidae) from eastern Brazil, with a synopsis of the Astyanax scabripinnis species complex. Neotropical Ichthyology 4 (1): 53-60.

Bertaco, V.A. \& L.R. Malabarba. 2001. Description of two new species of Astyanax (Teleostei: Characidae) from headwater streams of Southern Brazil, with comments on the " $A$. scabripinnis species complex". Ichthyological Exploration of Freshwaters 12 (3): 221-234.

Bizerril, C.R.S.F. 1997. Mudanças temporais de comunidades ícticas em uma bacia hidrográfica do leste brasileiro. Comunicações do Museu de Ciências e Tecnologia da PUCRS, Série Zoologia 10: 53-75.
Bollmann, H.A.; C. Carneiro \& E.S. Pegorini. 2005. Qualidade da água e dinâmica de nutrientes, p. 213-270. In: C.V. ANDreoli \& C. CARneiro (Eds). Gestão integrada de mananciais de abastecimento eutrofizados. Curitiba, Sanepar, Finep, 500p. Borror, D.J.; C.A. Triplehorn \& N.F. Johnson. 1989. An introduction to the study of insects. Fort Worth, Saunders College Publishing, 875p.

Buckup, P.A. 1999. Sistemática e Biogeografia de Peixes de Riachos, p. 91-138. In: E.P. Caramaschi; R. Mazzoni \& P.R. PeresNeto (Eds). Ecologia de Peixes de Riachos. Rio de Janeiro, PPGE-UFRJ, Série Oecologia Brasiliensis, vol. 6, 322p.

Casatti, L. 2002. Alimentação dos peixes em um riacho do Parque Estadual Morro do Diabo, bacia do alto rio Paraná, sudeste do Brasil. Biota Neotropica 2 (2): 1-14.

CASATTI, L. 2005. Fish assemblage structure in a first order stream, southeastern Brazil: Longitudinal distribution, seasonality, and microhabitat diversity. Biota Neotropica 5 (1): 1-9.

Casatti, L. \& R.M.C. Castro. 1998. A fish community of the São Francisco river headwaters riffles, southeastern Brazil. Ichthyological Explorator of Freshwaters 9 (3): 229-242.

Castro, R.M.C. 1999. Evolução da ictiofauna de riachos sulamericanos: padrões gerais e possíveis processos causais, p. 139-155. In: E.P. Caramaschi; R. Mazzoni \& P.R. Perez-Neto (Eds). Ecologia de peixes de riachos. Rio de Janeiro, PPGEUFRJ, Série Oecologia Brasiliensis, vol. 6, 322p.

Castro, R.M.C. \& L. Casatti. 1997. The fish fauna from a small forest stream of the upper Paraná River basin, southeastern Brazil. Ichthyological Exploration of Freshwaters 7 (4): 337-352.

Castro, R.M.C.; L. Casatti; H.F. Santos; K.M. Ferreira; A.C. RibeiRo; R.C. Benine; G.Z.P. Dardis; A.L.A. Melo; R. Stopiglia; T.A. Abreu; F.A. Bockmann; M. Carvalho; F.Z. Gibran; F.C.T. Lima. 2003. Estrutura e composição da ictiofauna de riachos do rio Paranapanema, Sudeste e Sul do Brasil. Biota Neotropica 3 (1): 1-31.

Chapman, J.A.; L. Harman \& D.E. Samuel. 1977. Reproductive and physiological cycles in the cottontail complex in western Maryland and nearby West Virginia. Wildlife Monographs 56: 1-73.

Costa, W.J.E.M. 1987. Feeding habits of a fish community in a tropical coastal stream, rio Mato Grosso, Brazil. Studies on Neotropical Fauna and Environment 22 (3): 145-153.

Crowder, L.B. \& W.E. CoOper. 1982. Habitat structural complexity and the interaction between bluegills and their prey. Ecology 63 (6): 1802-1813.

Eingenmann, C.H. 1921. The American Characidae. Memoirs of the Museum of Comparative Zoology 43 (3): 209-310.

Eingenmann, C.H. 1927. The American Characidae. Memoirs of the Museum of Comparative Zoology 43 (4): 311-428.

Esteves, K.E. 1996. Feeding ecology of three Astyanax species (Characidae, Tetragonopterinae) from a floodplain lake of Mogi-Guaçu River, Paraná River Basin, Brazil. Environmental Biology of Fishes 46: 83-101. 
Esteves, K.E. \& P.M. GaletTi. 1995. Food partitioning among characids of a small brazilian floodplain lake from the Paraná river basin. Enviromental Biology of Fishes 42: 375-389.

Garavello, J.C.; C.S. Pavanelli \& H.I. SuzuKi. 1997. Caracterização da ictiofauna do rio Iguaçu, p. 61-84. In: A.A. AgostinHo \& L.C. Gomes (Eds). Reservatório de Segredo: bases ecológicas para o manejo. Maringá, EDUEM, 387p.

GILINSKY, E. 1984. The role of fish predation and spatial heterogeneity in determining benthic community structure. Ecology 65 (2): 455-468.

Gomes, A.L. \& F.P. Monteiro. 1955. Estudo da população total de peixes da represa da estação experimental de biologia e piscicultura, em Pirassununga, São Paulo. Revista de Biologia Marina 6 (1/3): 82-133.

Gotceitas, V. \& P. Colgan. 1989. Predator foraging success and habitat complexity: quantitative test of the threshold hypothesis. Oecologia 80: 158-166.

GrEENBERG, L.A. 1991. Habitat use and feeding behavior of thirteen species of benthic stream fishes. Environmental Biology of Fishes 31: 389-401.

Gurgel, H.C.B. 1995. Estrutura da população de Metynnis $c f$. roosevelti Eigenmann, 1915 (Characidae, Myleinae) da Lagoa Redonda, Nízia Floresta, Rio Grande do Norte - Brasil. Boletim do Instituto de Pesca 22 (1): 61-71.

Hahn, N.S.; A.A. Agostinho; L.C. Gomes \& L.M. Bini. 1998. Estrutura trófica da ictiofauna do reservatório de Itaipu (ParanáBrasil) nos primeiros anos de formação. Interciência 23 (5): 299-305.

Harper, D.; J. Mekotova; S. Hulme; J. White \& J. Hall. 1997. Habitat heterogeneity and aquatic invertebrate diversity in floddplain forests. Global Ecology and Biogeography Letters 6: 275285.

Hynes, H.B.N 1950. The food of fish-water sticklebacks (Gasteronotus aculeatus and Pygosteus pungitius), with a review of methods used in studies of the food of fishes. Journal of Animal Ecology 19: 36-57.

Hyslop, E.J. 1980. Stomach contents analisys - a review of methods and their application. Journal of Fish Biology 17: 411-429.

Ingenito, L.F.S.; L.F.S. Duboc \& V. AbilhoA. 2004. Contribuição ao conhecimento da ictiofauna da bacia do alto rio Iguaçu, Paraná, Brasil. Arquivos de Ciências Veterinárias e Zoologia da UNIPAR 7 (1): 23-36.

Johnson, B.L. \& C.A. Jennings. 1998. Habitat Associations of Small Fishes around Islands in the Upper Mississippi River. North American Journal of Fisheries Management 18: 327-336.

KaWAKami, E. \& G. Vazzoler. 1980. Método gráfico e estimativa de índice alimentar aplicado no estudo de alimentação de peixes. Boletim do Instituto Oceanográfico 29 (2): 205207.

Le CREN, E.D. 1951. The length-weigth relationship and seasonal cycle in gonad weigh and condition in the perch (Perca fluviatilis). Journal of Animal Ecology 20: 201-219.
Lemes, E.M. \& V. Garutti. 2002. Ecologia da ictiofauna de um córrego de cabeceira da bacia do alto rio Paraná, Brasil. Iheringia, Série Zoologia, 92 (3): 69-78.

Lowe-McConnelL, R.H. 1975. Fish communities in tropical freshwater: their distribution, ecology and evolution. London, Longman, 337p.

LOWE-Mcconnell, R.H. 1999. Estudos ecológicos de comunidades de peixes tropicais. São Paulo, EDUSP, 535p.

Matthews, W.J. 1982. Small fish community structure in Ozark streams: structured assembly patterns or random abundance of species? American Midland Naturalist 107 (1): 42-54.

Matthews, W.J. 1998. Patterns in freshwater fish ecology. New York, Chapman and Hall, 756p.

Mazzoni, R. \& C.F. Rezende. 2003. Seasonal diet shift in a Tetragonopterinae (Osteichthyes, Characidae) from Ubatiba river, RJ, Brazil. Brazilian Journal of Biology 63 (1): 69-74.

Meffe, G.K. \& A.L. Sheldon. 1988. The influence of habitat structure on fish assemblage composition in southeastern blackwater streams. American Midland Naturalist 120 (2): 225-239.

Melo, F.A.G. 2001. Revisão taxonômica das espécies do gênero Astyanax Baird \& Girard, 1854, (Teleostei: Characiformes: Characidae) da região da Serra dos Órgãos. Arquivos do Museu Nacional 59: 1-46.

Moreira Filho, O. \& L.A. Bertollo. 1991. Astyanax scabripinnis (Pisces: Characidae), a species complex. Revista Brasileira de Genética 14 (2): 331-357.

Nomura, H. 1975. Fecundidade, maturação sexual e índice gonadossomático de lambaris do gênero Astyanax Baird \& Girard, 1854 (Osteichthyes, Characidae) relacionados com fatores ambientais. Revista Brasileira de Biologia 34 (4): 775-798.

Orsi, M.L.; E.D. Carvalho \& F. Foresti. 2004. Biologia populacional de Astyanax altiparanae Garutti \& Britski (Teleostei, Characidae) do médio rio Paranapanema, Paraná, Brasil. Revista Brasileira de Zoologia 21 (2): 207-218.

Oyakawa, O.T.; A. Akama; K.C. Mautari; J.C. Nolasco. 2006. Peixes de riachos da Mata Atlântica nas Unidades de Conservação do Vale do Rio Ribeira de Iguape no Estado de São Paulo. São Paulo, Neotrópica, 201p.

Peres Neto, P.R.; C.R.S.F. BizerRil \& R. Iglesias. 1995. An overview of some aspects of river ecology: a case study on fish assemblages distribution in an eastern Brazilian coastal river. Oecologia Brasiliensis 1: 317-334.

PÉREZ, G.R. 1988. Guía para el estudio de los macroinvertebrados acuáticos del Departamento de Antioquia. Bogotá, Universidad de Antioquia, Editorial Presencia, 217p.

Rezende, C.F. \& R. Mazzoni. 2006. Disponibilidade e uso de recursos alóctones por Bryconamericus microcephalus (MirandaRibeiro) (Actinopterygii, Characidae), no córrego Andorinha, Ilha Grande, Rio de Janeiro, Brasil Revista Brasileira de Zoologia 23 (1): 218-222.

Rozas, L.P. \& W.E. Odum. 1988. Occupation of submerged aquatic 
vegetation by fishes: testing the roles of food and refuge. Oecologia 77: 101-106.

Sabino, J. \& R.M.C. Castro. 1990. Alimentação, período de atividade e uso de espaço dos peixes de um riacho da Floresta Atlântica (Sudeste do Brasil). Revista Brasileira de Biologia 50: 23-36.

SAbINO, J. \& J. Zuanon. 1998. A stream fish assemblage in Central Amazonia: distribution, activity patterns and feeding behavior. Ichthyological Exploration of Freshwaters 8 (3): 201-210.

SANTOS, E.P. 1978. Dinâmica de populações aplicada a pesca e piscicultura. São Paulo, Edusp, 129p.

Schifino, L.C.; C.B. Fialho \& J.R. Verani. 1998. Reproductive aspects of Cyphocharax voga (Hensel) from Custódias Lagoon, Rio Grande do Sul, Brazil (Characiformes, Curimatidae). Revista Brasileira de Zoologia 15 (3): 767-773.

ShibatTa, O.A.; M.L. ORSI; S.T. Bennemann \& A.T. Silva-SouZA. 2002. Diversidade e distribuição de peixes na bacia do rio Tibagi, p. 403-423. In: M.E. Medri; E. Bianchini; O.A. Shibatta \& J.A. Pimenta (Eds). A bacia do rio Tibagi. Londrina, UEL, 595p.

Severi, W. \& A.A.M. Cordeiro. 1994. Catálogo de peixes da bacia do rio Iguaçu. Curitiba, IAP, GTZ, 128p.

Suzuki, H.I. \& A.A. Agostinho. 1997. Reprodução de peixes do reservatório de Segredo, p. 163-182. In: A.A. AgostinHo \& L.C. GoMEs (Eds). Reservatório de Segredo: bases ecológicas para o manejo. Maringá, EDUEM, 387p.

Takeuti, D.F.; J.R. Verani; J.M.R. Aranha \& M.S. Menezes. 1999. Population structure and condition factor of Pseudotothyris obtusa (Hypoptopomatinae) from three coastal streams in southern Brazil. Brazilian Archives of Biology and Technology 42 (4): 397-403.

TAYLOR, C.M. 1996. Abundance and distribution within a guild of benthic stream fishes: local processes and regional patterns. Freshwater Biology 36: 385-396.

UIEDA, V.S. 1984. Ocorrência e distribuição dos peixes em um riacho de água doce. Revista Brasileira de Biologia 44 (2):
203-213.

Vazzoler, A.E.A.M. 1981. Manual de Métodos para Estudos Biológicos de Populações de Peixes, Reprodução e Crescimento. Brasília, CNPq, 106p.

VAZzoler, A.E.A.M. 1996. Biologia da reprodução de peixes telósteos: teoria e prática. Maringá, Eduem, 169p.

Vazzoler, A.E.A.M. \& N.A. Menezes. 1992. Síntese dos conhecimentos sobre o comportamento reprodutivo dos Characiformes da América do Sul (Teleostei, Ostariophysi). Revista Brasileira de Biologia 52 (4): 626-640.

Veregue, A.M.L. \& M.L. Orsi. 2003. Biologia reprodutiva de Astyanax scabripinnis paranae (Eigenmann) (Ostheichthyes, Characidae) no Ribeirão das Marrecas, bacia do rio Tibagi, Paraná. Revista Brasileira de Zoologia 20: 97-105.

VIEIRA, S. 1980. Introdução à bioestatística. Rio de Janeiro, Campus, 196p.

VIEIRA, S. 2003. Bioestatística: tópicos avançados. Rio de Janeiro, Campus, 212p.

Vilella, F.S.; F.G. Becker \& S.M. Hartz. 2002. Diet of Astyanax species (Teleostei, Characidae) in an Atlantic forest river in Southern Brazil. Brazilian Archives of Biology and Technology 45 (2): 223-232.

Vono, V. \& F.A.R. BARBosa. 2001. Habitats and littoral zone fish community structure of two natural lakes in Southeast Brazil. Environmental Biology of Fishes 64: 1-9.

Weatherley, A.H. \& H.S. Gill. 1987. The biology of fish growth. London, Academic Press, 443p.

Welcomme, R.L. 1969. The biology and ecology of the fishes of a small tropical stream. Journal of Zoology 158: 485-529.

Willner, G.R.; J.A. Chapman \& D. Pursley. 1979. Reproduction, physiological responses, food habits, and abundance of nutria on Maryland Marshes. Wildlife Monographs 65: 1-43.

Wootton, R.J. 1999. Ecology of teleost fishes. The Netherlands, Kluwer Academic Publishers, 386p.

ZAR, J.H. 1996. Biostatistical analysis. London, Prentice Hall, $662 \mathrm{p}$.

Recebido em 01.III.2007; aceito em 14.XI.2007. 